\title{
SOME INCLUSION THEOREMS
}

\author{
by I. J. MADDOX
}

(Received 15 June, 1963)

1. A number of inclusion theorems have been given in connection with methods of summation which include the Riesz method $(R, \lambda, \kappa)$. Lorentz [4, Theorem 10] gives necessary and sufficient conditions for a sequence to sequence regular matrix $A=\left(a_{n, v}\right)$ to be such that $A \supset(R, \lambda, 1) \dagger$. He imposes restrictions on the sequence $\left\{\lambda_{n}\right\}$, so that $A$ does not include all Riesz methods of order 1 . In Theorem 1 below, we generalize the Lorentz theorem by giving a condition without restriction on $\lambda_{n}$. If the matrix $A$ is a series to sequence or series to function regular matrix, there do not appear to be any results concerning the general inclusion

$$
A \supset(R, \lambda, \kappa) \text {. }
$$

However, when $A$ is the Riemann method $(\mathfrak{R}, \lambda, \mu)$, Russell [7], generalizing earlier results, has given sufficient conditions for $(\Re, \lambda, \mu) \supset(R, \lambda, \kappa)$. Our Theorem 2 gives necessary and sufficient conditions for $A \supset(R, \lambda, 1)$, where $A$ satisfies the condition $a_{n, v} \rightarrow 1(n \rightarrow \infty, v$ fixed). Thus Theorem 2 applies to any series to sequence regular matrix $A$. In Theorem 3 we give a further representation for matrices $A$ which include $(R, \lambda, 1)$, and finally make some remarks on the problem of characterizing matrices which include Riesz methods of any positive order $\kappa$.

2. We write $s_{n}$ for the $(n+1)$ th partial sum of the series $\sum a_{m}$, where $\sum$ without limits denotes $\sum_{0}^{\infty}$. If

$$
\omega^{-\kappa} A^{\kappa}(\omega)=\omega^{-\kappa} \sum_{\lambda_{v}<\omega}\left(\omega-\lambda_{v}\right)^{\kappa} a_{v} \rightarrow s \quad(\omega \rightarrow \infty),
$$

where $0 \leqq \lambda_{0}<\lambda_{1}<\ldots<\lambda_{n} \rightarrow \infty$, and $\kappa>0$, we say that $\sum a_{n}=s(R, \lambda, \kappa)$. If $\omega$ takes only the values $\lambda_{n+1}$ we have summability by discontinuous Riesz means $(\widetilde{R}, \lambda, \kappa)$. For $0<\kappa \leqq 1$, Jurkat [3, Satz 2] has shown that $(R, \lambda, \kappa)$ and $(\bar{R}, \lambda, \kappa)$ are equivalent. We use this equivalence for the case $\kappa=1$ in what follows. We shall write

$$
\sigma_{n}=\sum_{v=0}^{n}\left(1-\frac{\lambda_{v}}{\lambda_{n+1}}\right) a_{v},
$$

so that $\sum a_{n}=s(R, \lambda, 1)$ is equivalent to $\sigma_{n} \rightarrow s(n \rightarrow \infty)$. By $\Delta \lambda_{n}$ we shall mean $\lambda_{n}-\lambda_{n+1}$.

3. The following theorem generalizes Lorentz's theorem mentioned above.

THEOREM 1. Let $A=\left(a_{n, v}\right)$ be a sequence to sequence regular matrix. Then $A \supset(R, \lambda, 1)$ if and only if

$$
\sum_{v=0}^{\infty} \lambda_{v+1}\left|\Delta\left(\frac{a_{n, v}}{\Delta \lambda_{v}}\right)\right| \leqq M,
$$

where $M$ is a positive constant.

† For methods of summability $A, B$, we write $A \supset B$ if the $B$-summability of a sequence or series implies its $A$-summability to the same limit. 
Proof. For each fixed $n$,

$$
\sum_{v=0}^{p} a_{n, v} s_{v}=-\sigma_{p} \lambda_{p+1} \frac{a_{n, p}}{\Delta \lambda_{p}}-\sum_{v=0}^{p-1} \sigma_{v} \lambda_{v+1} \Delta\left(\frac{a_{n, v}}{\Delta \lambda_{v}}\right) .
$$

The right hand side of (1) converges as $p \rightarrow \infty$, whenever $\left\{\sigma_{n}\right\}$ converges, if and only if the wellknown Toeplitz conditions are satisfied:

$$
\begin{array}{r}
\lambda_{p+1}\left|\frac{a_{n, p}}{\Delta \lambda_{p}}\right|+\sum_{v=0}^{p-1} \lambda_{v+1}\left|\Delta\left(\frac{a_{n, v}}{\Delta \lambda_{v}}\right)\right| \leqq M_{n}, \\
\exists \lim _{p \rightarrow \infty}-\lambda_{v+1} \Delta\left(\frac{a_{n, v}}{\Delta \lambda_{v}}\right)=\alpha_{v}(n) \quad(v \text { fixed }), \\
\exists \lim _{p \rightarrow \infty}\left\{-\lambda_{p+1} \frac{a_{n, p}}{\Delta \lambda_{p}}-\sum_{v=0}^{p-1} \lambda_{v+1} \Delta\left(\frac{a_{n, v}}{\Delta \lambda_{v}}\right)\right\}=\alpha(n) .
\end{array}
$$

Now (2) is equivalent to

$$
\sum \lambda_{v+1}\left|\Delta\left(\frac{a_{n, v}}{\Delta \lambda_{v}}\right)\right| \leqq M_{n}
$$

For (2) clearly implies (5), and when (5) holds we see that $\sum \Delta\left(a_{n, v} / \Delta \lambda_{v}\right)$ converges, and so

$$
a_{n, v} / \Delta \lambda_{v} \rightarrow t \quad(v \rightarrow \infty)
$$

Since $A$ is regular, we must have $t=0$; for if $t \neq 0$, then $a_{n, v} \sim t \Delta \lambda_{v}$, so that

$$
\sum_{v=0}^{N} a_{n, v} \sim-t \lambda_{N+1} \quad(N \rightarrow \infty)
$$

which contradicts the convergence of $\sum a_{n, v}$. It now follows from (5) and (6) that, as $p \rightarrow \infty$,

$$
\lambda_{p+1}\left|\frac{a_{n, p}}{\Delta \lambda_{p}}\right| \leqq \sum_{\nu=p}^{\infty} \lambda_{\nu+1}\left|\Delta\left(\frac{a_{n, v}}{\Delta \lambda_{v}}\right)\right|=o(1) .
$$

Hence (5) implies (2). Condition (3) is automatically satisfied, and (4) is satisfied with

$$
\alpha(n)=-\sum_{\nu=0}^{\infty} \lambda_{v+1} \Delta\left(\frac{a_{n, v}}{\Delta \lambda_{v}}\right) .
$$

Thus, for each fixed $n$, letting $p \rightarrow \infty$ in (1) we obtain

$$
\sum_{v=0}^{\infty} a_{n, v} s_{v}=-\sum_{v=0}^{\infty} \lambda_{\nu+1} \Delta\left(\frac{a_{n, v}}{\Delta \lambda_{v}}\right) \sigma_{v},
$$


whenever $\left\{\sigma_{v}\right\}$ is convergent and (5) holds. The right hand side of (8) tends to $s$ whenever $\sigma_{n}$ tends to $s$ if and only if

$$
\begin{gathered}
\sum \lambda_{v+1}\left|\Delta\left(\frac{a_{n, v}}{\Delta \lambda_{v}}\right)\right| \leqq M, \\
\lambda_{v+1} \Delta\left(\frac{a_{n, v}}{\Delta \lambda_{v}}\right) \rightarrow 0 \quad(n \rightarrow \infty, v \text { fixed }), \\
-\sum \lambda_{v+1} \Delta\left(\frac{a_{n, v}}{\Delta \lambda_{v}}\right) \rightarrow 1 \quad(n \rightarrow \infty),
\end{gathered}
$$

where we do not need (11) if $s=0$. Hence (9) is a necessary condition for $A \supset(R, \lambda, 1)$. To show that it is also sufficient we note that $(10)$ is an immediate consequence of the regularity of $A$. Also, when $s \neq 0$, if we let $s_{v}=1$ (all $v$ ) and take $\lambda_{0}=0$, as we may without loss in generality, then $\sigma_{v}=1$ (all $v$ ), so that (8) gives

$$
-\sum_{v=0}^{\infty} \lambda_{v+1} \Delta\left(\frac{a_{n, v}}{\Delta \lambda_{v}}\right)=\sum_{v=0}^{\infty} a_{n, v}
$$

Hence (11) holds when $A$ is regular.

This proves the theorem.

We now give the inclusion theorem for a series to sequence matrix $A$.

THeOREM 2. Let $A=\left(a_{n, v}\right)$ satisfy $a_{n, v} \rightarrow 1(n \rightarrow \infty, v$ fixed $)$. Then $A \supset(R, \lambda, 1)$ if and only if

(i) $\left|a_{n, v}\right| \leqq H_{n} \Lambda_{v}^{-1}$, where $\Lambda_{v}=\frac{\lambda_{v+1}}{\left|\Delta \lambda_{v}\right|}$,

(ii) $\sum_{v=0}^{\infty} \lambda_{v+1}\left|\Delta\left(\frac{\Delta a_{n, v}}{\Delta \lambda_{v}}\right)\right| \leqq M$,

where $H_{n}$ is a positive number depending on $n$, and $M$ is a positive constant.

Proof. By a convergence factor theorem of Jurkat [2, Satz 1], $\sum a_{n, v} a_{v}$ converges for each fixed $n$, whenever $\sum a_{v}=s(R, \lambda, 1)$, if and only if

$$
\begin{gathered}
\left|a_{n, v}\right| \leqq H_{n} \Lambda_{v}^{-1}, \\
\sum \lambda_{v+1}\left|\Delta\left(\frac{\Delta a_{n, v}}{\Delta \lambda_{v}}\right)\right| \leqq M_{n} .
\end{gathered}
$$

Jurkat gives the superfluous condition $\Delta a_{n, v} / \Delta \lambda_{v}=o(1)(v \rightarrow \infty, n$ fixed), which can be deduced from (12) and (13) by an argument of the type used in the proof of Theorem 1. It then follows easily from (13) that in fact

$$
\lambda_{v+1}\left|\frac{\Delta a_{n, v}}{\Delta \lambda_{v}}\right| \leqq M_{n} .
$$


Now, for each fixed $n$,

$$
\sum_{v=0}^{p} a_{n, v} a_{v}=s \alpha_{n}-\sum_{v=0}^{p-1} \lambda_{\nu+1} \Delta\left(\frac{\Delta a_{n, v}}{\Delta \lambda_{v}}\right)\left(\sigma_{v}-s\right)-\lambda_{p+1} \frac{a_{n, p}}{\Delta \lambda_{p}}\left(\sigma_{p}-s\right)+\lambda_{p} \frac{a_{n, p+1}}{\Delta \lambda_{p}}\left(\sigma_{p-1}-s\right),
$$

where $\alpha_{n}=\left(\lambda_{1} a_{n, 0}-\lambda_{0} a_{n, 1}\right) /\left(\lambda_{1}-\lambda_{0}\right)$. Hence, provided that (12) and (13) hold and $\sigma_{v} \rightarrow s$,

$$
\sum_{\nu=0}^{\infty} a_{n, v} a_{v}=s \alpha_{n}-\sum_{\nu=0}^{\infty} \lambda_{\nu+1} \Delta\left(\frac{\Delta a_{n, v}}{\Delta \lambda_{v}}\right)\left(\sigma_{v}-s\right),
$$

since

and, in view of (14),

$$
\lambda_{p+1}\left|\frac{a_{n, p}}{\Delta \lambda_{p}}\right| \leqq H_{n}
$$

$$
\lambda_{p} \frac{\left|a_{n, p+1}\right|}{\left|\Delta \lambda_{p}\right|} \leqq \lambda_{p} \frac{\left|\Delta a_{n, p}\right|}{\left|\Delta \lambda_{p}\right|}+\lambda_{p} \frac{\left|a_{n, p}\right|}{\left|\Delta \lambda_{p}\right|} \leqq M_{n}+H_{n} .
$$

Since $\alpha_{n} \rightarrow 1$ by the hypothesis that $a_{n, v} \rightarrow 1(n \rightarrow \infty, v$ fixed), the right hand side of (15) tends to $s$ whenever $\sigma_{v} \rightarrow s$ if and only if (ii) of the theorem holds and

$$
\lambda_{v+1} \Delta\left(\frac{\Delta a_{n, v}}{\Delta \lambda_{v}}\right) \rightarrow 0 \quad(n \rightarrow \infty, v \text { fixed }) .
$$

But this condition is automatically satisfied by the hypothesis on $a_{n, v}$.

The proof is complete.

The next theorem gives an alternative representation for the matrices $A$ of Theorem 2 .

Theorem 3. Let $A$ be a series to sequence matrix with $a_{n, v} \rightarrow 1(n \rightarrow \infty, v$ fixed $)$, and suppose that $\Lambda_{n}=\lambda_{n+1} /\left(\lambda_{n+1}-\lambda_{n}\right)$ is unbounded. $†$ Then $A \supset(R, \lambda, 1)$ if and only if

(i) $\left|a_{n, v}\right| \leqq H_{n} \Lambda_{v}^{-1}$,

(ii) there exists a sequence of functions $\left\{g_{n}(u)\right\}$, defined for $u \geqq \lambda_{0}$, such that

$$
a_{n, v}=\int_{\lambda_{v}}^{\infty}\left(u-\lambda_{v}\right) d g_{n}(u) \text {, with } \int_{\lambda_{0}}^{\infty} u\left|d g_{n}(u)\right| \leqq M,
$$

where $H_{n}$ is a positive number depending on $n$ and $M$ is a positive constant.

Proof. Necessary and sufficient conditions for $\sum a_{n, v} a_{v}$ to converge for each fixed $n$, whenever $\sum a_{v}$ is summable $(R, \lambda, \kappa), \kappa \geqq 0$, have been given by Maddox [5, Theorem A]. In the case $\kappa=1$, these conditions are (i) of Theorem 3, and (ii) with $M$ replaced by $M_{n}$. For $\kappa \geqq 0$.

$\dagger$ This involves no real loss in generality, since, if $\Lambda_{n}$ is bounded, $(R, \lambda, \kappa)$ is equivalent to convergence for 
the sufficiency part of Theorem 3 we need only show that (ii) of Theorem 2 is implied by (ii) of Theorem 3. This follows, for we have

$$
\Delta\left(\frac{\Delta a_{n, v}}{\Delta \lambda_{v}}\right)=-\int_{\lambda_{v+1}}^{\lambda_{v+2}} d g_{n}(u)+\Delta \int_{\lambda_{v}}^{\lambda_{v+1}}\left(\frac{u-\lambda_{v}}{\Delta \lambda_{v}}\right) d g_{n}(u) .
$$

Hence, since $\lambda_{v+1}\left(u-\lambda_{v}\right) \leqq u\left(\lambda_{v+1}-\lambda_{v}\right)$ for $\lambda_{v} \leqq u \leqq \lambda_{v+1}$,

$$
\sum \lambda_{v+1}\left|\Delta\left(\frac{\Delta a_{n, v}}{\Delta \lambda_{v}}\right)\right| \leqq 3 M \text {. }
$$

We must now prove the necessity of the condition

$$
\int_{\lambda_{0}}^{\infty} u\left|d g_{n}(u)\right| \leqq M
$$

in (ii). This is included in the following lemma, which gives necessary conditions for the inclusion $A \supset(R, \lambda, \kappa), \kappa>0$.

Lemma 1. Let $A=\left(a_{n, v}\right)$ be a series to sequence matrix, and suppose that $\dagger^{\dagger}$

(a) $0<a \leqq \frac{\Delta \lambda_{n}}{\Delta \lambda_{n-1}} \leqq A \quad(a, A$ constants),

(b) $\lambda_{n+1} / \lambda_{n}$ decreases to 1 .

Necessary conditions that $A \supset(R, \lambda, \kappa)(\kappa>0)$ are

(i) $\left|a_{n, v}\right| \leqq H_{n} \Lambda_{v}^{-\kappa}$, where $\Lambda_{v}=\frac{\lambda_{v+1}}{\left|\Delta \lambda_{v}\right|}$,

(ii) there exists a sequence of functions $\left\{g_{n}(u)\right\}$, defined for $u \geqq \lambda_{0}$, and a number $M>0$, independent of $n$, such that

$$
a_{n, v}=\int_{\lambda_{v}}^{\infty}\left(u-\lambda_{v}\right)^{\kappa} d g_{n}(u), \text { with } \int_{\lambda_{0}}^{\infty} u^{\kappa}\left|d g_{n}(u)\right| \leqq M .
$$

When $0<\kappa \leqq 1$, the restrictions $(a)$, (b) may be removed, provided that $\Lambda_{n}$ is unbounded.

Proof. We first recall some of the properties of the function spaces associated with Riesz summability. For detailed definitions, reference should be made to Peyerimhoff [6]. If $C_{c}$ is the normed vector space of functions $x(t)$, continuous for $t \geqq 0$, which tend to limits as $t \rightarrow \infty$, with norm $\|x\|=\overline{\mathrm{bd}}|x(t)|(t \geqq 0)$, then

$$
f(x)=\alpha \lim _{t \rightarrow \infty} x(t)+\int_{0}^{\infty} x(t) d h(t), \text { with } \int_{0}^{\infty}|d h(t)|<\infty,
$$

$\dagger$ We write $\lambda \in \Lambda$ when $(a)$ and $(b)$ hold. 
where $\alpha$ is a constant, is a continuous linear functional in $C_{c}$ and each continuous linear functional in $C_{c}$ may be represented in this way. The norm of $f$ is given by

$$
\|f\|=|\alpha|+\int_{0}^{\infty}|d h(t)| \text {. }
$$

In what follows $R_{\lambda \kappa}(r, c)$ denotes the space of sequences $\left\{a_{v}\right\}$ such that $\sum a_{v}$ is summable $(R, \lambda, \kappa)$. With the norm defined by

$$
\left\|\left\{a_{v}\right\}\right\|=\overline{\mathrm{bd}} \frac{\left|A^{\kappa}(t)\right|}{t^{\kappa}} \quad(t \geqq 0),
$$

$R_{\lambda \kappa}(r, c)$ is a Banach space $[6$, p. 46].

Peyerimhoff $\left[6\right.$, p. 48] used (16) to give the general continuous linear functional $f\left(\left\{a_{v}\right\}\right)$ in the space $R_{\lambda \kappa}(r, c)$ :

$$
f\left(\left\{a_{v}\right\}\right)=\alpha \lim _{t \rightarrow \infty} \frac{A^{\kappa}(t)}{t^{\kappa}}+\int_{\lambda_{0}}^{\infty} \frac{A^{\kappa}(t)}{t^{\kappa}} d h(t) ; \int_{\lambda_{0}}^{\infty}|d h(t)|<\infty .
$$

The Hahn-Banach extension theorem was used to establish (18), and since the norm can be preserved under extension, we have, by (17),

If we write

$$
\left\|f\left(\left\{a_{v}\right\}\right)\right\|=|\alpha|+\int_{\lambda_{0}}^{\infty}|d h(t)| .
$$

$$
g(t)=-\int_{t}^{\infty} u^{-\kappa} d h(u) \quad\left(t \geqq \lambda_{0}\right),
$$

we bring (18), (19) to the form

$$
\begin{gathered}
f\left(\left\{a_{v}\right\}\right)=\alpha \lim _{t \rightarrow \infty} \frac{A^{\kappa}(t)}{t^{\kappa}}+\int_{\lambda_{0}}^{\infty} A^{\kappa}(u) d g(u) ; \int_{\lambda_{0}}^{\infty} u^{\kappa}|d g(u)|<\infty, \\
\\
\left\|f\left(\left\{a_{v}\right\}\right)\right\|=|\alpha|+\int_{\lambda_{0}}^{\infty} u^{\kappa}|d g(u)| .
\end{gathered}
$$

We obtain from (20) a necessary condition for $\sum a_{n, v} a_{v}$ to converge for each fixed $n$, whenever $\sum a_{v}$ is summable $(R, \lambda, \kappa)$, by taking $\dagger f_{n}\left(\left\{a_{v}\right\}\right)=\sum a_{n, v} a_{v}$ in (20), with $a_{p}=1$, $a_{v}=0(v \neq p)$. Thus

$$
a_{n, p}=\alpha+\int_{\lambda_{p}}^{\infty}\left(u-\lambda_{p}\right)^{\kappa} d g_{n}(u)
$$

The necessity of condition (i) follows from Theorem A in [5]. If we combine (22) and (i) of the lemma, we see that $\alpha=0$. Now it was proved in the sufficiency part of Theorem A of [5] that

$$
\sum_{\nu=0}^{\infty} a_{n, v} a_{\nu}=\int_{\lambda_{0}}^{\infty} A^{\kappa}(u) d g_{n}(u)
$$

$+f_{n}$ is a continuous linear functional in $R_{\lambda \kappa}(r, c)$ (see [6], p. 29, II). 
whenever $\sum a_{v}$ is summable $(R, \lambda, \kappa)$. Thus we have a sequence of continuous linear functionals $\left\{\sum a_{n, v} a_{v}\right\}$ in $R_{\lambda \kappa}(r, c)$, which by (21) with $\alpha=0$, and (23), have norms

$$
\left\|\sum_{v=0}^{\infty} a_{n, v} a_{v}\right\|=\int_{\lambda_{0}}^{\infty} u^{\kappa}\left|d g_{n}(u)\right|
$$

If $\sum a_{v}$ is summable $A$, then

$$
\varlimsup\left|\sum_{v=0}^{\infty} a_{n, v} a_{v}\right|<\infty
$$

By the Banach-Steinhaus theorem (Banach [1, Theorem 5, p. 80]) it follows from (24) and (25) that $\left\{\int_{\lambda_{0}}^{\infty} u^{\kappa}\left|d g_{n}(u)\right|\right\}$ is bounded.

The lemma is now proved.

Theorem 3 and Lemma 1 suggest that there is a general inclusion theorem of the following type:

Let $A$ be a series to sequence regular matrix and suppose that $\lambda \in \Lambda$. Then $A \supset(R, \lambda, \kappa)$ $(\kappa>0)$ if and only if

(i) $\left|a_{n, v}\right| \leqq H_{n} \Lambda_{v}^{-\kappa}$, where $\Lambda_{v}=\frac{\lambda_{v+1}}{\left|\Delta \lambda_{v}\right|}$,

(ii) $A$ is of the form

$$
a_{n, v}=\int_{\lambda_{v}}^{\infty}\left(u-\lambda_{v}\right)^{\kappa} d g_{n}(u) \text { with } \int_{\lambda_{0}}^{\infty} u^{\kappa}\left|d g_{n}(u)\right| \leqq M .
$$

The necessity is included in Lemma 1 , and the sufficiency, in the case $\kappa=1$, in Theorem 3. I have not been able to prove that the conditions are sufficient in any other case, although it seems likely that they will be. If this theorem could be proved it would generalize many inclusion theorems for Riesz means, and in particular, for $\lambda_{n}=n$, would give an inclusion theorem for Cesàro means.

4. I wish to thank the referee for a number of helpful comments, which have clarified some of the proofs.

\section{REFERENCES}

1. S. Banach, Théorie des opérations linéaires (New York, 1955).

2. W. Jurkat, Uber Konvergenzfaktoren bei Rieszschen Mitteln, Math. Zeit. 54 (1951), 262-271.

3. W. Jurkat, Über Rieszsche Mittel mit unstetigem Parameter, Math. Zeit., 55 (1951), 8-12. 
4. G. G. Lorentz, Riesz methods of summation and orthogonal series, Trans. Royal Soc. of Canada (3) XLV (1951), 19-32.

5. I. J. Maddox, Convergence and summability factors for Riesz means, Proc. London Math. Soc. (3) 12 (1962), 345-366.

6. A. Peyerimhoff, Konvergenz und Summierbarkeitsfaktoren, Math. Zeit. 55 (1951), $23-54$.

7. D. C. Russell, On Riesz and Riemann summability, Trans. American Math. Soc. 104 (1962), 383-391.

THE UNIVERSITY

BIRMINGHAM 